Supporting Information for

\title{
Artificial Sub-60 Millivolts/Decade Switching in a Metal-Insulator-Metal-Insulator- Semiconductor Transistor without Ferroelectric Component
}

\author{
Peng $W u^{1,2 *}$, Joerg Appenzeller ${ }^{1,2 *}$ \\ ${ }^{1}$ Birck Nanotechnology Center, Purdue University, West Lafayette, Indiana 47907, USA \\ ${ }^{2}$ Department of Electrical and Computer Engineering, Purdue University, West Lafayette, Indiana 47907, \\ USA \\ *E-mail:wu936@purdue.edu, appenzeller@purdue.edu
}




\section{Determination of capacitance and resistance components in the RC network model}

Fig. S1a shows a schematic of the capacitance and resistance components in the $\mathrm{WS}_{2} \mathrm{MIMIS}$ device. The overlapping area of internal gate and gate is $A_{\text {int }-G}=1 \mu \mathrm{m}^{2}$ and the area of the contact pad for the internal gate is $A_{\text {pad }}=50 \mu \mathrm{m} \times 50 \mu \mathrm{m}=2500 \mu \mathrm{m}^{2}$, respectively. The device area of the $\mathrm{WS}_{2}$ FET is $A_{\text {device }}=1 \mu \mathrm{m}^{2}$. The capacitances can be calculated:

$$
\begin{gathered}
C_{\mathrm{int}-G}=\frac{\varepsilon_{\mathrm{AlO}_{x}} \varepsilon_{0} A_{\text {int }-G}}{t_{\mathrm{AlO}_{x}}}=\frac{7 \times 8.854 \times 10^{-12} \times 1 \times\left(10^{-6}\right)^{2}}{8 \times 10^{-9}}=7.75 \times 10^{-15} \mathrm{~F} \\
C_{p}=\frac{\varepsilon_{\mathrm{SiO}_{2}} \varepsilon_{0} A_{\mathrm{pad}}}{t_{\mathrm{SiO}_{2}}}=\frac{3.9 \times 8.854 \times 10^{-12} \times\left(50 \times 10^{-6}\right)^{2}}{90 \times 10^{-9}}=9.59 \times 10^{-13} \mathrm{~F} \\
C_{\mathrm{ox}}=\frac{\varepsilon_{\mathrm{HfO}_{2}} \varepsilon_{0} A_{\text {device }}}{t_{\mathrm{HfO}_{2}}}=\frac{13 \times 8.854 \times 10^{-12} \times\left(1 \times 10^{-6}\right)^{2}}{7 \times 10^{-9}}=1.64 \times 10^{-14} \mathrm{~F}
\end{gathered}
$$

The calculation of $C_{q}$ can be found in Supporting Section 3, yet since it's in series with $C_{o x}$, the total capacitance $C_{M O S}$ is smaller than $C_{o x}$. Note that $C_{p}$ is $\sim 60$ times larger than $C_{o x}$ for $90 \mathrm{~nm} \mathrm{SiO}_{2}$. Therefore, we can ignore $C_{M O S}$ when simulating the $V_{\text {int }}$ response. As we illustrated in Figure 4 in the main text, if $C_{p}$ could be eliminated, the effect of the voltage-dependent $C_{M O S}$ would become relevant and can lead to a steep switching even for a measurement with a constant scan rate. Yet with $\mathrm{Si} / \mathrm{SiO}_{2}$ substrates, it is challenging to achieve thicker than $\sim 500 \mathrm{~nm} \mathrm{SiO}_{2}$ using typical thermal oxidation methods, which would only reduce $C_{p}$ to $\sim 1 / 6$ of its current value, and $C_{p}$ would still be $10 \mathrm{x}$ larger than $C_{o x}$. Therefore, $C_{p}$ could not be eliminated by using a substrate with thicker $\mathrm{SiO}_{2}$ dielectric. One possible solution is to switch to an insulating substrate, such as sapphire, yet this would make identifying flakes almost impossible.

Next, the resistance value for $R$ is determined. Fig. S1b shows the leakage current $I_{\text {leak }}$ in the $\mathrm{AlO}_{\mathrm{x}}$ capacitor as a function of voltage difference $V_{\text {diff }}=V_{G}-V_{\text {int }}$, which clearly exhibits a non-linear behavior and different $R$ values can be extracted at different $V_{\text {diff }}$-values. In addition, due to inaccuracies and noises in the measurement, there is a significant uncertainty associated with the extracted $R$ value, especially when 
the current is small. Therefore, we have adopted an indirect method of extracting $R$ by fitting the measured $V_{\text {int }}-V_{G}$ curve, as shown in Fig. S1c. We have derived in the main text that, in the steady state:

$$
V_{G}-V_{\text {int }}=R C_{p} \frac{\mathrm{d} V_{G}}{\mathrm{~d} t}
$$

Therefore, different $R$ values lead to different $V_{G^{-}} V_{\text {int }}$ values and thus different hysteresis voltage values in the bidirectional $V_{G}$ scan. As clearly shown in Fig. S1c, $R=600 \mathrm{G} \Omega$ gives the best fit to the experimental data, while other values either underestimate or overestimate the hysteresis voltage. The good fitting also indicates that using a fixed value for $R$ is a good approximation despite the non-linear $I_{\text {leak }}-V_{\text {diff }}$ characteristics. Fig. S1d shows the simulated $V_{i n t}-V_{G}$ as a function of time using the parameter $R=600$ $\mathrm{G} \Omega$. The voltage difference is below $0.21 \mathrm{~V}$ during the $V_{G}$ voltage scan, and from the $I_{\text {leak }}-V_{\text {diff }}$ characteristics in Supporting Fig $1 \mathrm{~b}$, we can roughly estimate $R \approx 900 \mathrm{G} \Omega$ at $V_{G^{-}} V_{\text {int }}=-0.2 \mathrm{~V}$, which is in acceptable agreement with the extracted $R=600 \mathrm{G} \Omega$. 
a

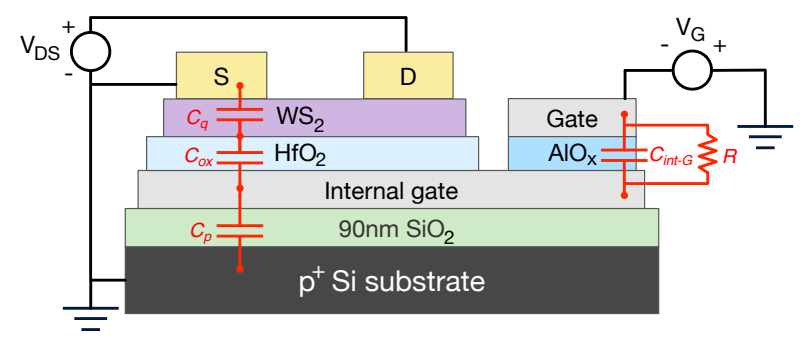

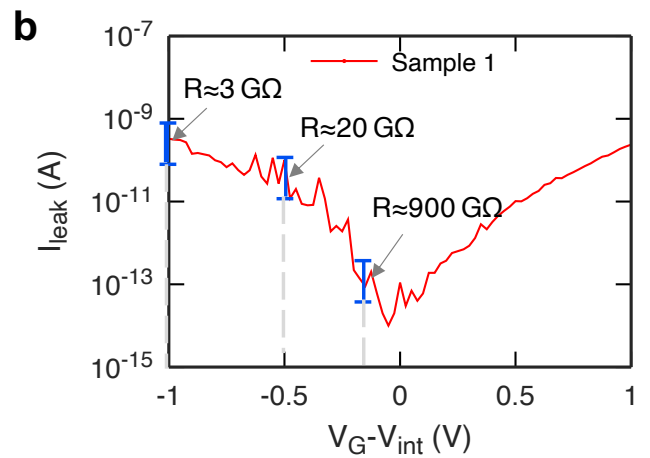

C

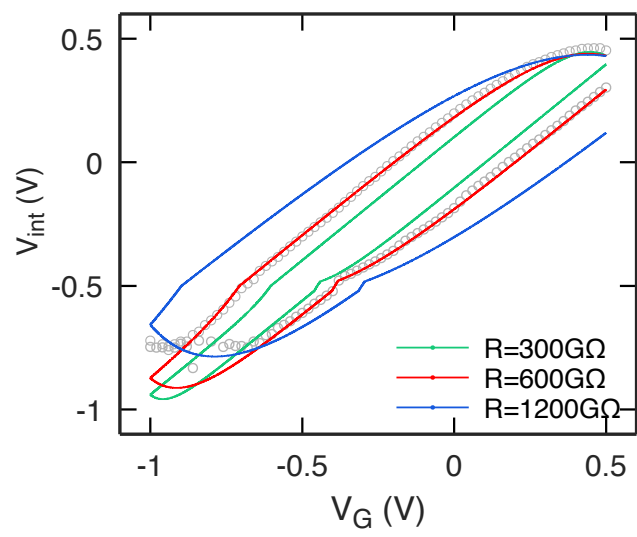

d

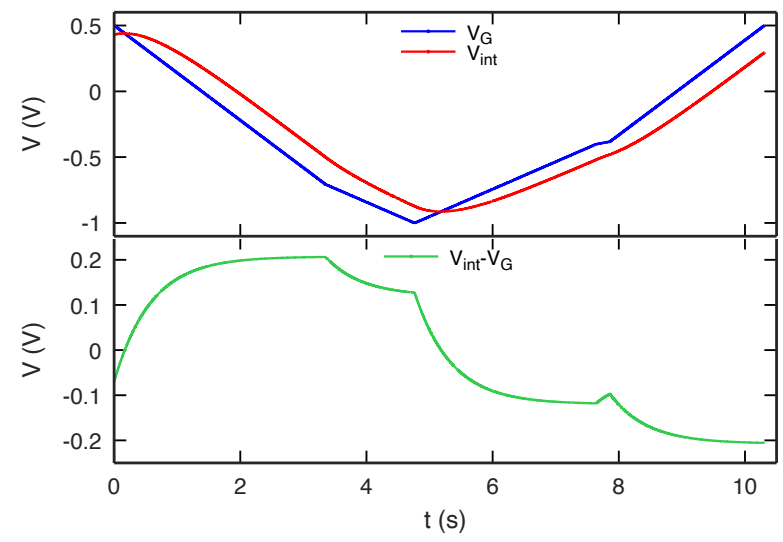

Figure S1. Determination of capacitance and resistance in the RC network model. (a), Schematic of capacitance and resistance components in the $\mathrm{WS}_{2}$ MIMIS FET. (b) Leakage current $I_{\text {leak }}$ in the $\mathrm{AlO}_{\mathrm{x}}$ capacitor versus $V_{G}-V_{\text {int }}$, showing non-linear characteristics and different $R$ values at different $V_{\text {diff. }}$ (c) Simulated $V_{\text {int }}-V_{G}$ for different $R$ values. Line: simulation. Symbol: experiment. (d) Simulated $V_{G}, V_{\text {int }}$ and $V_{\text {int }}-V_{G}$ versus time $t$.

\section{Extraction of scan rates from experiment}

Supporting Video 1 shows a video of a researcher performing an $I_{D}-V_{G}$ scan of the $\mathrm{WS}_{2}$ MIMIS FET on the Agilent 4156C parameter analyzer (a single frame of the video is shown in Fig. S2a as an example).

The measured $I_{D}-V_{G}$ characteristics are shown in Fig. S2b. Note that the measurement was performed $\sim 3$ months after the initial measurement (Figure $2 \mathrm{~d}$ in the main text), and the characteristics have drifted slightly compared to the initial characterization. In particular, the hysteresis voltage had become larger, which indicates an increase in the resistance $R$ of the $\mathrm{AlO}_{\mathrm{x}}$ capacitor according to our analysis from the previous section. However, the main features, such as sub- $60 \mathrm{mV} /$ decade switching and anti-clockwise hysteresis, are still retained in the new measurement. We speculate that the resistance increase may be related to a decrease in oxygen vacancies while the $\mathrm{AlO}_{\mathrm{x}}$ film is exposed to air. It has been shown in Refs. 
[1-4] that aluminum forms a natural oxide layer that acts as a decent gate dielectric after sufficiently long air exposure. Yet in our device, we limit the air exposure time of $\mathrm{Al}$ to $\sim 15$ minutes before capping it with another metal layer. As a result, the $\mathrm{AlO}_{\mathrm{x}}$ film may be sub-stoichiometric and likely contains oxygen vacancies, which contribute to the leaky behavior. It has also been reported in Ref. [1] that after $\mathrm{AlO}_{\mathrm{x}}$ dielectric breaks down, it can "self-heal" upon further oxidation in air, which is in line with our speculation.

From the video, we extract the gate voltage $V_{G}$ as a function of time $t$ for $V_{D S}=0.4 \mathrm{~V}$, as shown by the red dots in Fig. S2c. It is clear that two distinct scan rates of $0.36 \mathrm{~V} / \mathrm{s}$ ("fast") and $0.208 \mathrm{~V} / \mathrm{s}$ ("slow") can be extracted from the measured $V_{G}-t$ curve, which correspond to $I_{D}$ above and below $1 \mathrm{nA}(0.6 \mathrm{nA} / \mu \mathrm{m})$, respectively. The values for the "fast" and "slow" scans are applied in the simulation for the $V_{\text {int }}$ response in the main text. In the forward scan direction $(0.5 \mathrm{~V}$ to $-1 \mathrm{~V})$, sub-60 $\mathrm{mV} /$ decade switching is observed when the scan rate transitions from "fast" to "slow" at $I_{D}=1 \mathrm{nA}$ (green shaded area). In the backward scan (-1 V to $0.5 \mathrm{~V})$, two sub-60 $\mathrm{mV} /$ decade switching events are observed, one at $I_{D}=1 \mathrm{nA}$ and another at $I_{D}=100 \mathrm{nA}$, which correspond to the overhead time associated with two measurement range changes, as shown by the orange shaded areas in Fig. S2c. Interestingly, the second sub- $60 \mathrm{mV} / \mathrm{decade}$ event at $I_{D}$ $=100 \mathrm{nA}$ was not observed in the initial measurement. The cause for the difference is still under investigation, which we speculate might be from some minor changes in the settings of the parameter analyzer in the initial measurement and the new measurement, despite our effort of making them the same. 
a
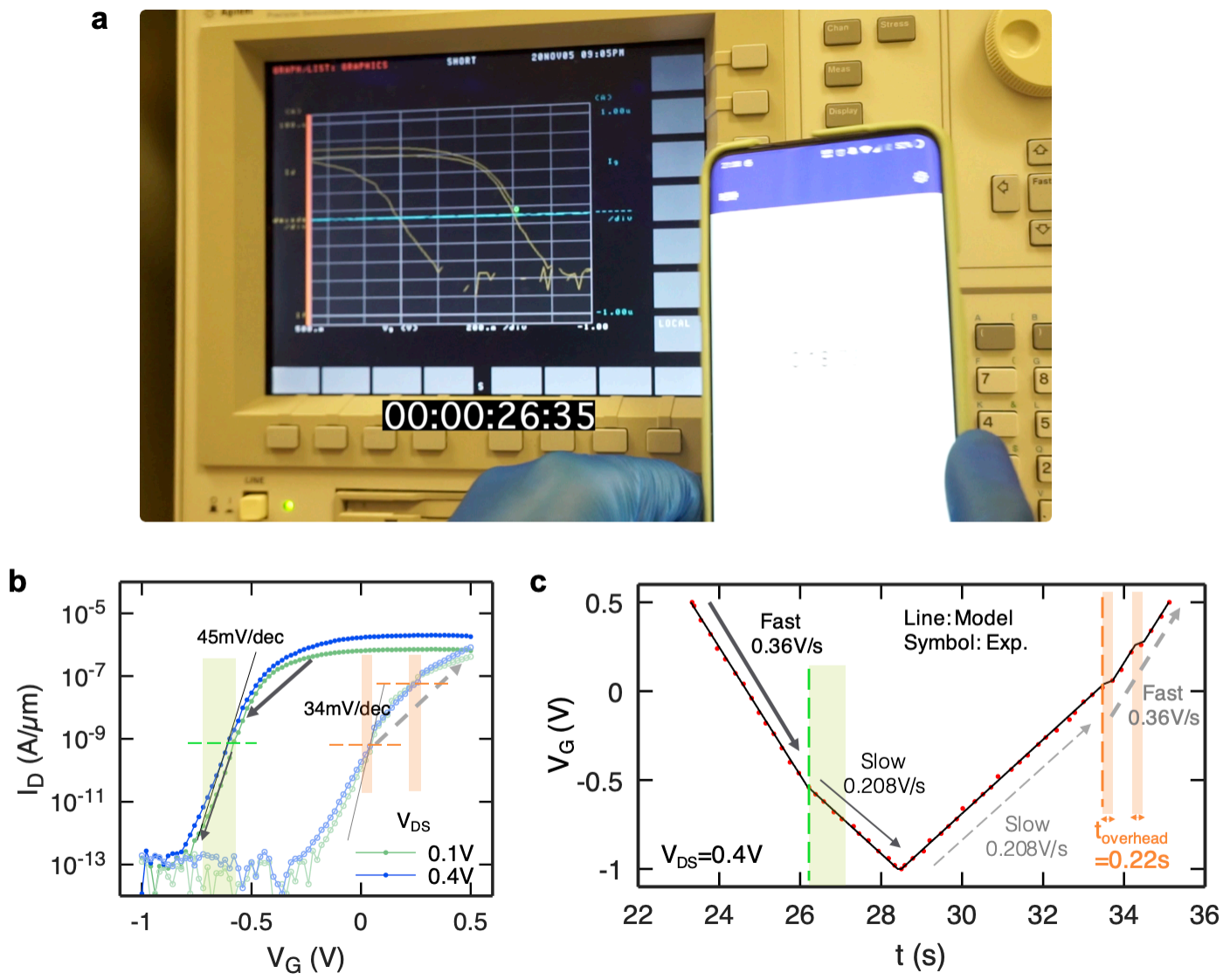

Figure S2. Extraction of scan rates from experiment. (a) A single frame from the video of performing an $I_{D}$ $-V_{G}$ scan on the WS 2 MIMIS FET. (b) Measured $I_{D}-V_{G}$ characteristics. (c) $V_{G}$ as a function of time, showing two scan rates at different current levels and overhead time in the backward scan direction.

\section{Calculation of quantum capacitance $C_{q}$}

Fig. S3a shows the schematic of the capacitance model for the $\mathrm{WS}_{2}$ internal FET. The quantum capacitance $C_{q}$ of $2 \mathrm{D} \mathrm{WS}_{2}$ channel is given by:

$$
C_{q}=\frac{\partial Q_{\mathrm{ch}}}{\partial \psi_{S}}=q^{2} \operatorname{DOS}_{2 \mathrm{D}} f\left(E_{C}\right) A_{\text {device }}=q^{2} \frac{g_{C} m_{e}^{*}}{\pi \hbar^{2}} \frac{1}{1+\exp \left(\frac{E_{C}-E_{F}}{k_{B} T}\right)} A_{\text {device }}
$$

Eq. (5) describes the dependence of $C_{q}$ on the relative position of $E_{C}$ and Fermi level $E_{F}$, or equivalently the surface potential $\psi_{S}$. In order to calculate the $V_{\text {int }}$ dependence of $C_{q}$, we need to determine the relation of $\psi_{S}$ and $V_{i n t}$, which is given by:

$$
\frac{\mathrm{d} \psi_{S}}{\mathrm{~d} V_{\mathrm{int}}}=\frac{C_{\mathrm{ox}}}{C_{\mathrm{ox}}+C_{q}}
$$




$$
\psi_{S}=\frac{\Phi_{\mathrm{SB}, n}+E_{F}-E_{C}}{q}
$$

Note that we have chosen flat-band condition as the zero point for the potential $\psi_{S}$, i.e., $\psi_{S}=0$ when $V_{\text {int }}=$ $V_{F B}$. For multilayer $\mathrm{WS}_{2}$ in this study, we have:

$$
g_{C}=6, m_{e}^{*}=0.22 m_{0}
$$

The only missing parameters are the flat-band voltage $V_{F B}$ and electron Schottky barrier height $\Phi_{\mathrm{SB} . n}$. We extract these parameters by fitting experimental $I_{D}-V_{\text {int }}$ data with a Landauer model, ${ }^{5,6}$ as shown in Fig. S3b. Using the Landauer model, we extract the parameters:

$$
V_{F B}=-0.46 \mathrm{~V}, \Phi_{\mathrm{SB}, \mathrm{n}}=0.33 \mathrm{eV},
$$

and the screening length for Schottky barrier injection $\lambda=2.6 \mathrm{~nm}$. Notice that above $V_{t h}$ (the extraction of its value will be discussed next), the simulated curve from Landauer model starts to deviate from experimental data. This is because the Landauer approach is based on a ballistic transport picture, while above $V_{t h}$, electrons start to populate the channel and a diffusive picture should be adopted for the long channel device $(L \approx 0.6 \mu \mathrm{m})$ in this study. However, the fitting for the data below $V_{t h}$, where scattering can be ignored, is already sufficient to extract the critical parameters and we did not try to simulate the on-state of the device quantitatively.

Having determined the values of $V_{F B}$ and $\Phi_{\mathrm{SB}, \mathrm{n}}$, we calculate $\psi_{S}$ as a function of $V_{\text {int }}$ based on Eqs. (6-7), as shown in Fig. S3c. One can see that $\psi_{S}$ is following $V_{\text {int }}$ one-to-one below $V_{t h}=-0.26 \mathrm{~V}$. Above $V_{t h}$, channel charges $Q_{c h}$ become significant and $\psi_{S}$ starts to saturate and deviate from the one-to-one relation.

Finally, we calculate $C_{q}$ as a function of $\psi_{S}$ based on Eq. (5), as shown by the blue line in Fig. S3d. One would expect $C_{q}$ to show up as a step function of $\psi_{S}$ at $T=0 \mathrm{~K}$, yet at finite temperatures, the transition becomes more gradual due to thermal broadening. By applying the $\psi_{S}-V_{\text {int }}$ relation shown in Fig. S3c, we can determine $C_{q}$ as a function of $V_{i n t}$, as shown by the red line in Fig. S3d. Note that $C_{q, \max } \gg C_{o x}$, 
which indicates $C_{M O S}$, the series capacitance of $C_{o x}$ and $C_{q}$, will quickly saturate to $C_{o x}$ once $V_{\text {int }}$ is above $V_{t h}$, as shown in Figure $4 \mathrm{~b}$ in the main text.

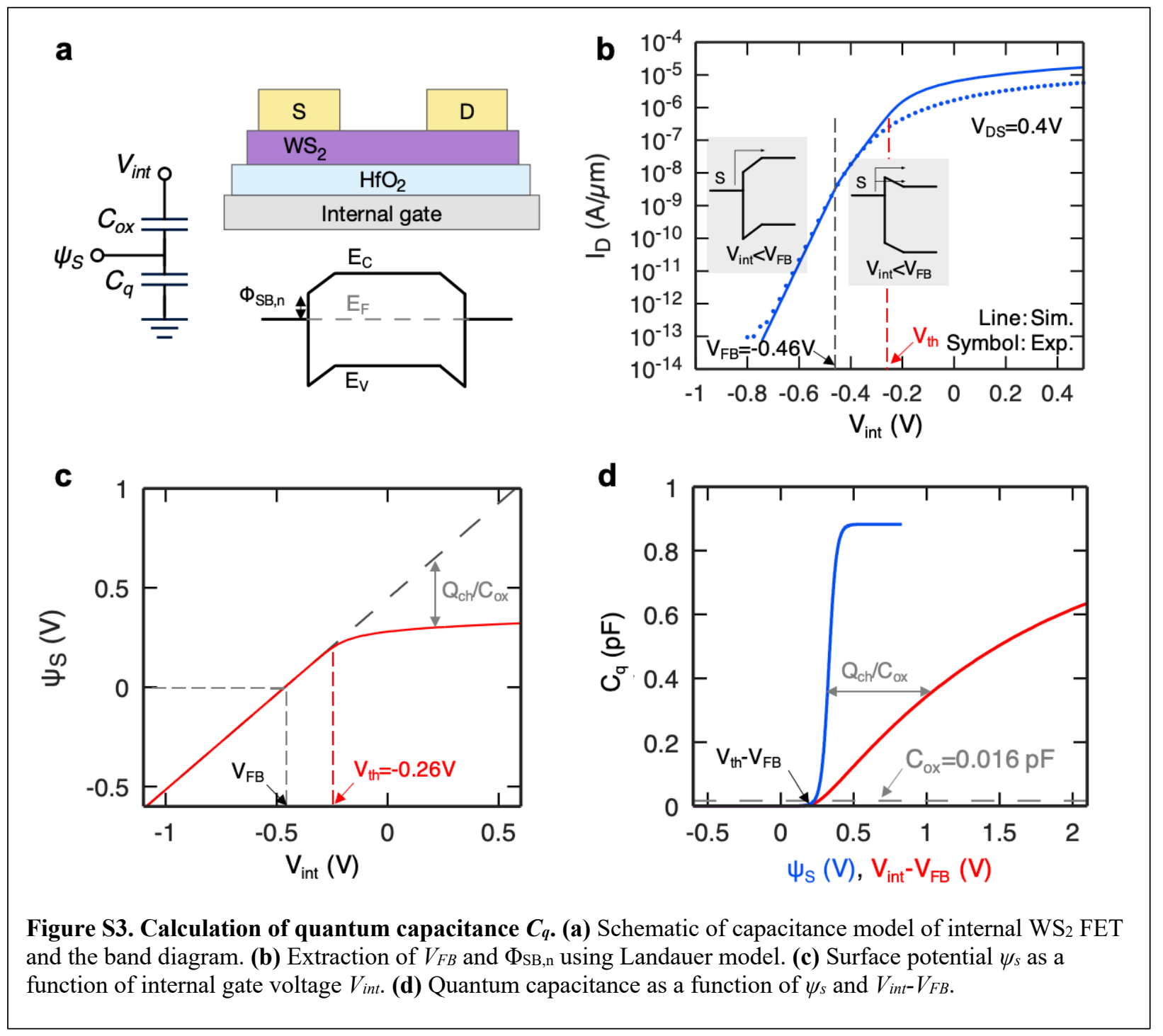

\section{Direct measurement of waveform of gate voltage}

Fig. S4a shows the schematic of measurement setup for measuring the waveforms of test signals. A parameter analyzer (Agilent 4156C, denoted as "parameter analyzer \#1") was used to generate the voltage signals to the device under test (DUT) and measure the currents, using source-measurement units (SMUs) 1, 2 and 3 for source, drain and gate (or internal gate, if measuring the internal FET), respectively. In order to measure the waveform of the gate voltage without disturbing the measurement of the actual 
DUT, we used an additional SMU 4 in the synchronous sweep measurement mode, which effectively "copies" the voltage output from SMU 3, i.e., the gate voltage, and used an additional parameter analyzer (Agilent 4156C, denoted as "parameter analyzer \#2") in sampling measurement mode to record the waveform of the "copied" gate voltage from parameter analyzer \#1.

Next, we measure the device transfer characteristics and record the waveforms of the gate voltage during the measurement. Note that we have performed these measurements on a different device from the same batch as the device described in the main text. Fig. S4b shows the measured $I_{D}-V_{\text {int }}$ characteristics of the internal FET. The internal FET exhibits an SS-value of $61 \mathrm{mV} /$ decade and negligible hysteresis. Fig. S4c shows the measured waveform of $V_{\text {int }}$ as a function of time during the $I_{D}-V_{\text {int }}$ measurement, from which one can observe the bidirectional sweeps of $V_{\text {int }}$ at two different drain voltages and two distinctive scan rates, "fast" and "slow".

Fig. S4d shows the measured $I_{D}-V_{G}$ characteristics of the MIMIS FET, from which one can observe an anticlockwise hysteresis and sub-60 mV/decade switching in both sweep directions. Fig. S4e shows the measured waveform of $V_{G}$ as a function of time during the $I_{D}-V_{G}$ measurement. In the forward scan direction $(1 \mathrm{~V}$ to $-1 \mathrm{~V})$, one can observe a transition from "fast" scan rate to "slow" scan rate at $V_{G}=-0.7$ $\mathrm{V}$, exactly the gate voltage at which the sub-60 mV/decade switching is observed. In the backward scan direction $(-1 \mathrm{~V}$ to $1 \mathrm{~V})$, with the directly measured waveform of $V_{G}$, one can see that there exist several plateau regions due to overhead times associated with measurement range changes. From the enlarged views of the $I_{D}-V_{G}$ characteristics and measured $V_{G}$ waveform shown in Fig. S4f and $4 \mathrm{~g}$, respectively, it becomes very apparent that there are four "kinks" in the $I_{D}-V_{G}$ characteristics and four corresponding plateau regions in the $V_{G}$ waveform, which are associated with the measurement range changes at $10^{-9} \mathrm{~A}$, $10^{-8} \mathrm{~A}, 10^{-7} \mathrm{~A}$ and $10^{-6} \mathrm{~A}$, respectively. Note that the overhead time at $V_{G}=-0.2 \mathrm{~V}$ [labeled as "(1)"] is about $0.24 \mathrm{~s}$, which is associated with the measurement range change at $10^{-9} \mathrm{~A}$, where the transition from 
"slow" to "fast" scan rate occurs, and the value is in agreement with $t_{\text {overhead }}=0.22 \mathrm{~s}$ that was used in the simulation in the main text.

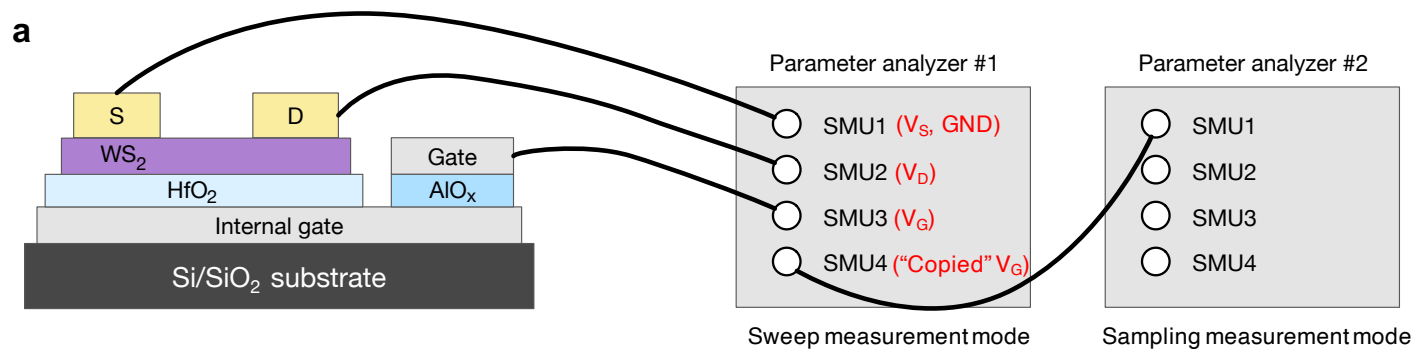

b

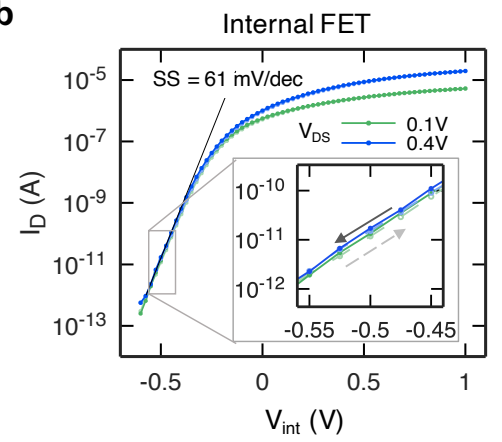

d

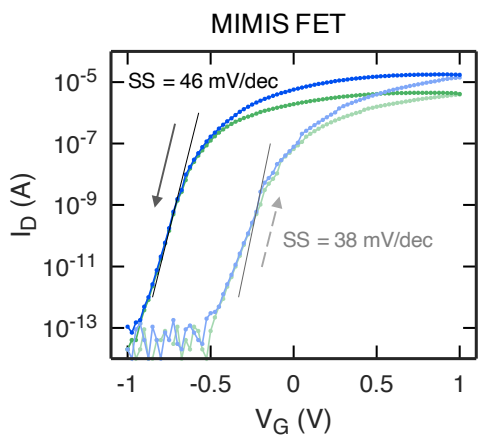

f

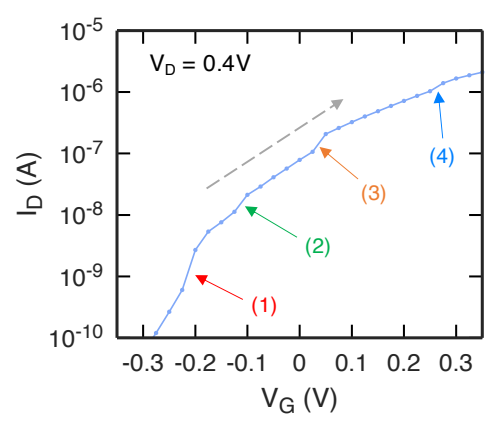

C Measurement start

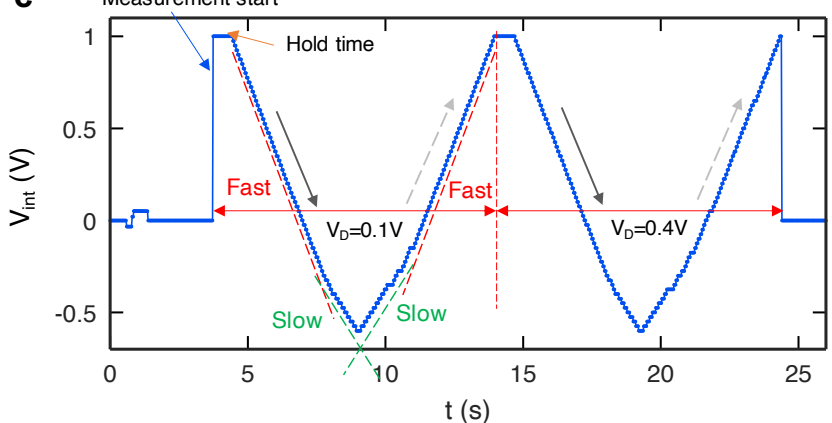

e
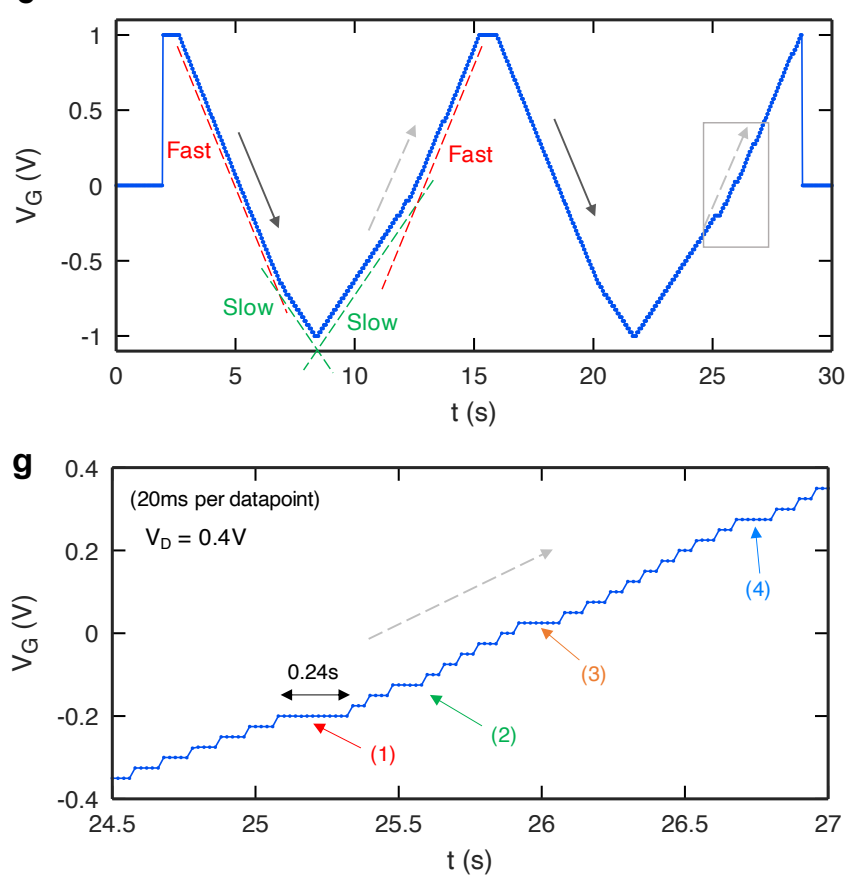

Figure S4. Measurement of waveforms of gate voltage. (a) Schematic of measurement setup for measuring testing signals using the sampling measurement of the parameter analyzer. (b) $I_{D}-V_{\text {int }}$ characteristics of the internal FET. (c) Measured waveform of $V_{\text {int }}$ as a function of time. (d) $I_{D}-V_{G}$ characteristics of the MIMIS FET. (e) Measured waveform of $V_{G}$ as a function of time. (f) Enlarged view of $I_{D}-V_{G}$ characteristics of the MIMIS FET in the backward scan direction at $V_{D}=0.4 \mathrm{~V}$. (g) Enlarged view of measured waveform of $V_{G}$ as a function of time. 


\section{Measurement of internal FET with different scan rates and hold time}

We have measured the internal FET with different scan rates and hole time $t_{\text {hold }}$ to highlight that the near ideal SS and small hysteresis are independent to measurement setup. Figures S5a and S5b show the measured $I_{D}-V_{\text {int }}$ characteristics of the internal FET and waveform of $V_{\text {int }}$ during the measurement with $t_{\text {delay }}=0 \mathrm{~s}$ and $t_{\text {hold }}=0.5 \mathrm{~s}$. A near ideal slope of $61 \mathrm{mV} /$ decade and negligible hysteresis are observed in the $I_{D}-V_{\text {int }}$ characteristics. The resulting scan rates are $0.39 \mathrm{~V} / \mathrm{s}$ ("fast") and $0.236 \mathrm{~V} / \mathrm{s}$ ("slow") for current levels above and below $10^{-9} \mathrm{~A}$, respectively. Note that the values are different from $0.36 \mathrm{~V} / \mathrm{s}$ ("fast") and $0.208 \mathrm{~V} / \mathrm{s}$ ("slow") that were used in the main text, since a different voltage step size (0.025 $\mathrm{V}$, instead of $0.02 \mathrm{~V}$ in the main text) were used here unintentionally. Figures S5c and S5d show the measured $I_{D}-V_{\text {int }}$ characteristics of the internal FET and waveform of $V_{\text {int }}$ during the measurement with $t_{\text {delay }}=0.5 \mathrm{~s}$ and $t_{\text {hold }}=5 \mathrm{~s}$. A near ideal slope of $60 \mathrm{mV} /$ decade and negligible hysteresis are observed in the $I_{D}-V_{\text {int }}$ characteristics, despite the much slower scan rate of $0.044 \mathrm{~V} / \mathrm{s}$. Note that the $I_{D}-V_{\text {int }}$ characteristics are shifted by about $-0.1 \mathrm{~V}$ in Fig. S5c compared with Fig. S5a, possibly due to trapped charges induced by repeated measurements. Also note that a few outlier points in the backward scan direction at $V_{D}=0.4 \mathrm{~V}$ in Fig. S5c are a result of an unstable contact between the probe tips and the metal pads for source/drain electrodes, which we found to be occurring in prolonged measurements ( $>100 \mathrm{~s})$, possibly due to vibrations loosening the contact. 

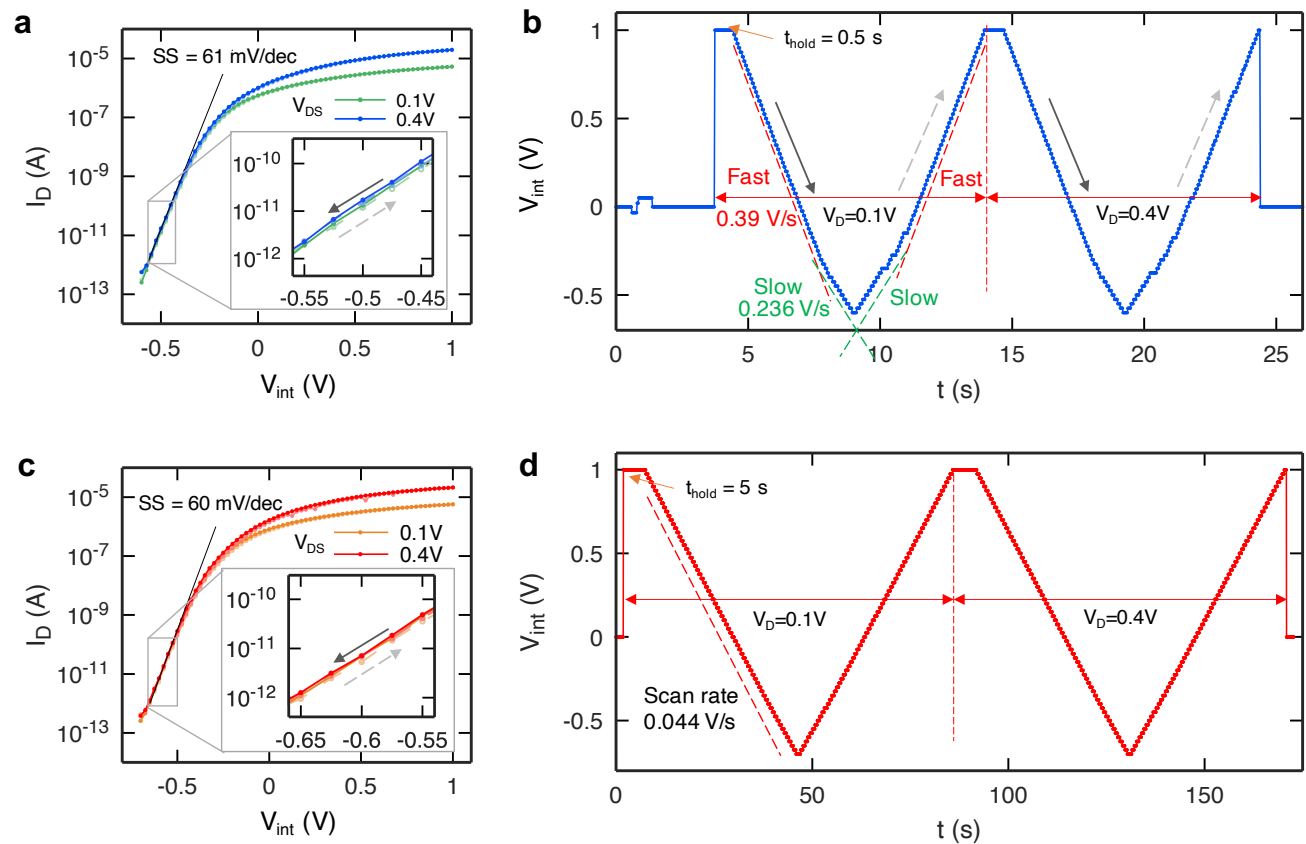

Figure S5. Measurement of internal FET with different scan rates. (a) $I_{D}-V_{\text {int }}$ characteristics of the internal FET with $t_{\text {delay }}=0 \mathrm{~s}$ and $t_{\text {hold }}=0.5 \mathrm{~s}$. (b) Measured waveform of $V_{\text {int }}$ as a function of time with $t_{\text {delay }}=0 \mathrm{~s}$ and $t_{\text {hold }}=0.5 \mathrm{~s}$. (c) $I_{D}-V_{\text {int }}$ characteristics of the internal FET with $t_{\text {delay }}=0.5 \mathrm{~s}$ and thold $=5 \mathrm{~s}$. (d) Measured waveform of $V_{\text {int }}$ as a function of time with $t_{\text {delay }}=0.5 \mathrm{~s}$ and $t_{\text {hold }}=5 \mathrm{~s}$.

\section{Measurement of MIMIS FET with constant gate voltage scan rate}

In the main text, we have attributed the sub-60 mV/decade switching in the MIMIS FET to varying scan rates of gate voltage $V_{G}$. Since the capacitance in the RC circuit model is dominated by the constant parasitic capacitance $C_{p}$, we expect no steep switching behavior for a constant $V_{G}$ scan rate. Here, we characterize the MIMIS FET with a constant $V_{G}$ scan rate to verify our theory.

Figures S6a and S6b show the measured $I_{D}-V_{G}$ characteristics of the MIMIS FET and waveform of $V_{G}$ during the measurement with $t_{\text {delay }}=0 \mathrm{~s}$ and $t_{\text {hold }}=0.5 \mathrm{~s}$, which leads to varying scan rates of $0.39 \mathrm{~V} / \mathrm{s}$ ("fast") and $0.236 \mathrm{~V} / \mathrm{s}$ ("slow") for current levels above and below $10^{-9} \mathrm{~A}$, respectively. As we have analyzed in the main text and Supporting Section 4, in the forward scan direction (1V to -1V), one can observe sub- $60 \mathrm{mV} /$ decade switching at $V_{G}=-0.7 \mathrm{~V}$, at which the scan rate changes from "fast" to "slow", and in the backward scan direction (-1V to 1V), steep switching can be observed when the auto- 
ranging of the parameter analyzer changes the measurement range, which leads to an overhead time.

Figures S6c and S6d show the measured $I_{D}-V_{G}$ characteristics of the MIMIS FET and waveform of $V_{G}$ during the measurement with $t_{\text {delay }}=0.5 \mathrm{~s}$ and $t_{\text {hold }}=0.5 \mathrm{~s}$, which leads to a constant scan rate of 0.044 V/s. As expected, no steep switching behaviors are observed in the transfer characteristics. Please note that this is an expected behavior due to a large, constant parasitic capacitance $C_{p}$ being dominant in the RC network model, and as we illustrated in Figure 4 in the main text, if $C_{p}$ could be eliminated, a steep switching behavior could still be observed even with a constant scan rate. As we have mentioned in Supporting Section 5, the outlier points in Fig. S6c are a result of unstable contact between the probe tips and the metal pads for source/drain electrodes, which we found to be occurring for prolonged measurements ( $>100 \mathrm{~s})$, possibly due to vibrations loosening the contact. Also note that a finite hysteresis still exists even for a constant scan rate in Fig. S6c, which is a result of the leaky dielectric, yet the hysteresis is much smaller due to a slower scan rate, which is in agreement with our calculation in Eq. (3) from the main text.
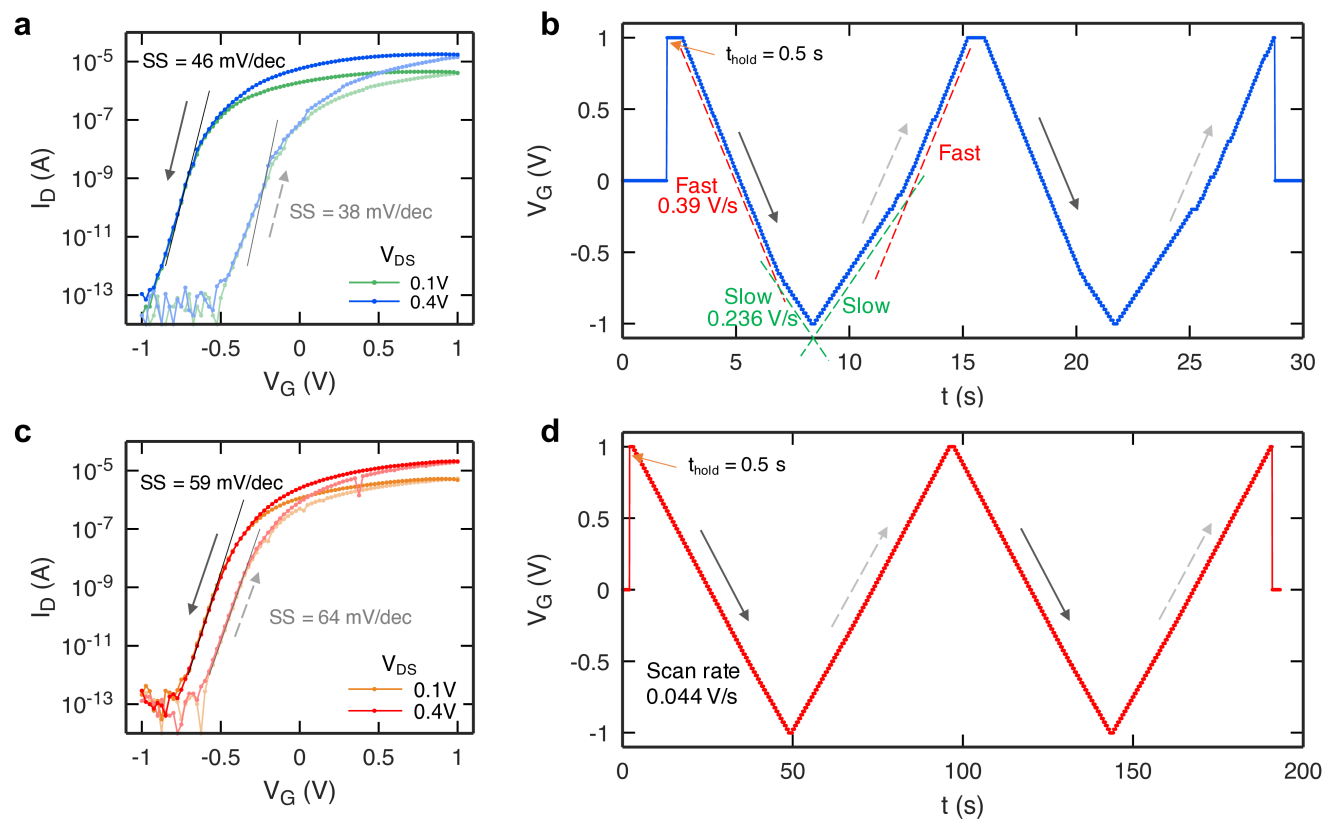

Figure S6. Measurement of MIMIS FET with different scan rates. (a) $I_{D}-V_{G}$ characteristics of the MIMIS FET with $t_{\text {delay }}=0 \mathrm{~s}$ and thold $=0.5 \mathrm{~s}$. (b) Measured waveform of $V_{G}$ as a function of time with $t_{\text {delay }}=0 \mathrm{~s}$ and $t_{\text {hold }}$ $=0.5 \mathrm{~s}$. (c) $I_{D}-V_{G}$ characteristics of the MIMIS FET with $t_{\text {delay }}=0.5 \mathrm{~s}$ and $t_{\text {hold }}=0.5 \mathrm{~s}$. (d) Measured waveform of $V_{G}$ as a function of time with $t_{\text {delay }}=0.5 \mathrm{~s}$ and $t_{\text {hold }}=0.5 \mathrm{~s}$. A constant scan rate is obtained. 


\section{Measurement of MIMIS FET with larger $\mathrm{AlO}_{\mathrm{x}}$ layer leakage resistance}

Fig. S7a shows the leakage currents of the $\mathrm{AlO}_{\mathrm{x}}$ capacitors as a function of applied voltage in two different samples, where Sample 1 denotes the device in the main text and Sample 4 denotes another device in the same batch that happens to have much lower leakage current. One can see that in this device, the leakage current is below the noise floor of the measurement $\left(\sim 10^{-13} \mathrm{~A}\right)$ when the applied voltage $V_{\text {diff }}=V_{G^{-}} V_{\text {int }}$ is below $\pm 1 \mathrm{~V}$. Fig. S7b shows the $I_{D}-V_{\text {int }}$ characteristics of the internal FET in Sample 4, in which near ideal SS and hysteresis-free characteristics are observed. Fig. S7c shows the $I_{D}-$ $V_{G}$ characteristics of the MIMIS FET in Sample 4 with different voltage scan ranges. When the scan range is $1 \mathrm{~V}$ to $-1.2 \mathrm{~V}$, the device cannot be turned off, which is distinctively different from the MIMIS FET in Sample 1, shown in Figure $2 \mathrm{~b}$ in the main text. When the scan range is extended to $1 \mathrm{~V}$ to $-1.4 \mathrm{~V}$, the OFF-current can reach a lower value of below $1 \mathrm{nA}$. Finally, when the scan range is extended to $1 \mathrm{~V}$ to $-1.6 \mathrm{~V}$, the device is able to turn off completely, and steep-slope switching are again observed in both scan directions. The $I_{D}-V_{G}$ characteristics of the MIMIS FET with different voltage scan ranges are compiled in a single plot in Fig. S7d, and combined with $I_{D}-V_{\text {int }}$ characteristics, we derive the $V_{\text {int }}-V_{G}$ relations under different $V_{G}$ scan ranges, as shown in Fig. S7e. From the $V_{\text {int }}-V_{G}$ plot, we can see that in the forward scan direction, $V_{\text {int }}$ remains almost unchanged at $0 \mathrm{~V}$ until $V_{G}$ reaches below $-1 \mathrm{~V}$, after which $I_{\text {leak }}$ starts to show up, allowing $V_{\text {int }}$ to follow $V_{G}$ while maintaining the voltage difference. The scan in the backward scan direction shows a similar behavior, although with $V_{\text {diff }}=V_{G^{-}} V_{\text {int }}$ changing its polarity. The $V_{G}$ scan window needs to be large enough to allow $V_{\text {diff, }}$, and thus $I_{\text {leak }}$, to be large enough in order to see the complete switching. Interestingly, this plot in some way resembles the minor and major $P-E$ loop scans in ferroelectric materials.

Another way to understand this observation is already discussed in the main text: "the $R C$ time constant needs to be comparable to the timescale of the $V_{G}$ scan in order to see the described effects." Note that the leaky $\mathrm{AlO}_{\mathrm{x}}$ film is a highly nonlinear resistor, which indicates that even if $R$ is too large under small $V_{\text {diff, }}$ under larger $V_{\text {diff, }} R$ may become smaller and fall into the range in which the $R C$ constant is comparable to 
the sweep time. In this interpretation, the $V_{G}$ scan window also needs to be large enough to allow a large enough $V_{\text {diff }}$ to make the RC time constant "compatible" with the sweep time.
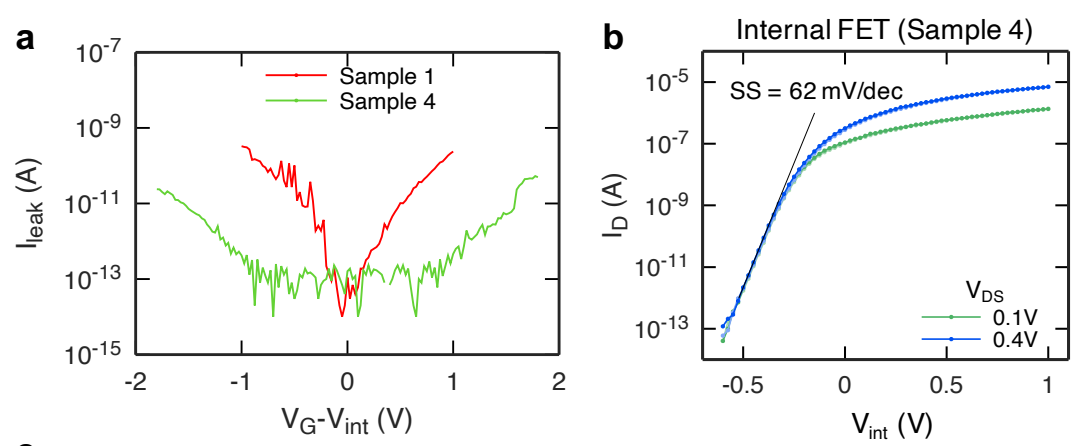

C

MIMIS FET (Sample 4)
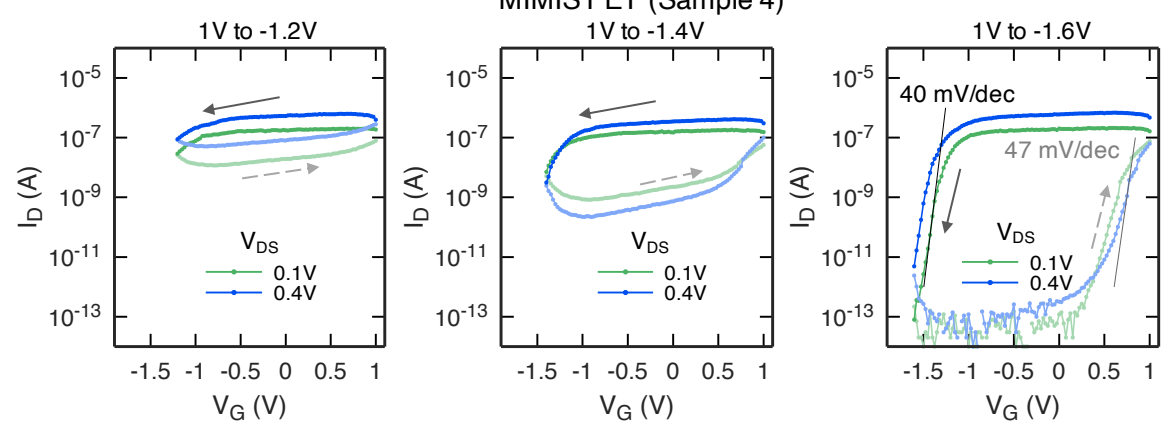

d
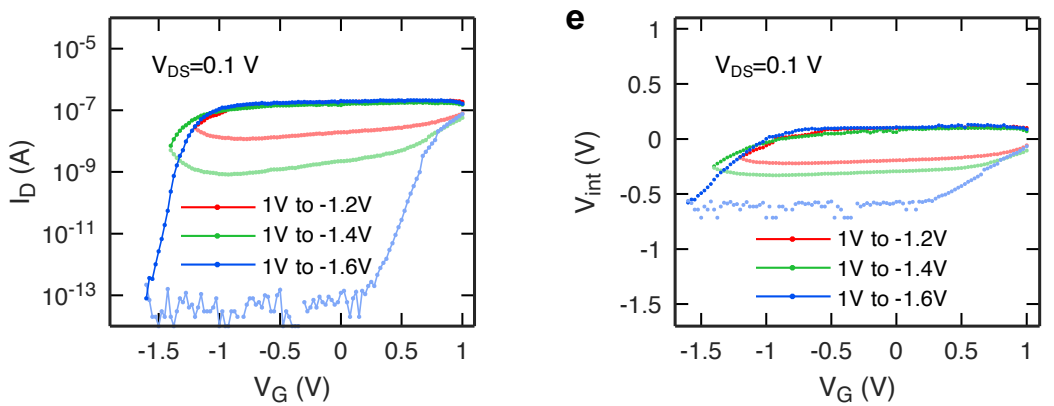

Figure S7. Measurement of MIMIS FET with large AlOx layer leakage resistance. (a) $I_{l e a k}-V_{\text {diff }}$ characteristics of the $\mathrm{AlO}_{x}$ capacitors in two different samples. (b) $I_{D}-V_{\text {int }}$ characteristics of the internal FET. (c) $I_{D}-V_{G}$ characteristics of the MIMIS FET with different voltage scan ranges. (d) A compilation of $I_{D}-V_{G}$ characteristics of the MIMIS FET with different voltage scan ranges. (e) Extracted $V_{\text {int }}$ as a function of $V_{G}$ during $I_{D}-V_{G}$ measurement with different voltage scan ranges.

\section{Characterization of the thin Ti layer as internal gate electrode}

In our device, a thin Ti layer was used as internal gate electrode. The reason for using a thin Ti layer is that it is well known that the evaporation process for depositing the $\mathrm{AlO}_{\mathrm{x}}$ layer has a poor step coverage, and we intentionally used a thin Ti layer to avoid an excessive leakage current at the side wall, as depicted in Fig. S8a. 
To address a possible concern about the resistance of the thin Ti layer being too large, we have characterized the conductivity of the thin Ti layer with a nominal thickness of $2 \mathrm{~nm}$, same as in our device, using a transfer length method (TLM) structure as shown in Fig. S8b. A 3- $\mu$ m-wide Ti stripe with $2 \mathrm{~nm}$ nominal thickness was deposited using e-beam evaporation and patterned using e-beam lithography and liftoff process, followed by patterning and deposition of $40 \mathrm{~nm}$ thick Ni contacts. Note that the Ti layer was exposed to air for $\sim 24$ hours before patterning and depositing the Ni metal leads, which is much longer than the typical air exposure time for the Ti gate layer in our device. Therefore, any possible oxidation of Ti from air exposure is already taken into consideration in our test. Fig. S8c shows the $I-V$ characteristics of Ti stripes with different lengths. The linear $I-V$ characteristics indicates Ohmic contacts. Fig. S8d shows the TLM measurement results, from which we can extract a contact resistance of $2 R_{C}=3.26 \mathrm{k} \Omega$ and a sheet resistance of $R_{\text {sheet }}=2.95 \mathrm{k} \Omega / \mathrm{sq}$. Note that the resistance of the leaky $\mathrm{AlO}_{\mathrm{x}}$ layer is $\sim 600 \mathrm{G} \Omega$, which is more than seven orders of magnitude higher than the resistance of the Ti layer. Therefore, the voltage drop within the internal gate electrode can be ignored.

In addition, we have performed AFM scans on the " $2 \mathrm{nm"} \mathrm{Ti} \mathrm{layer,} \mathrm{as} \mathrm{shown} \mathrm{in} \mathrm{Fig.} \mathrm{S8e,} \mathrm{from} \mathrm{which} \mathrm{we}$ found that the actual thickness of the Ti layer is $5 \mathrm{~nm}$. This could be attributed to the crystal monitor in our evaporator not being well-calibrated for depositing thin films at a low evaporation rate $(0.5 \AA / \mathrm{s})$. A thickness change after Ti oxidizes in air could be another reason. 


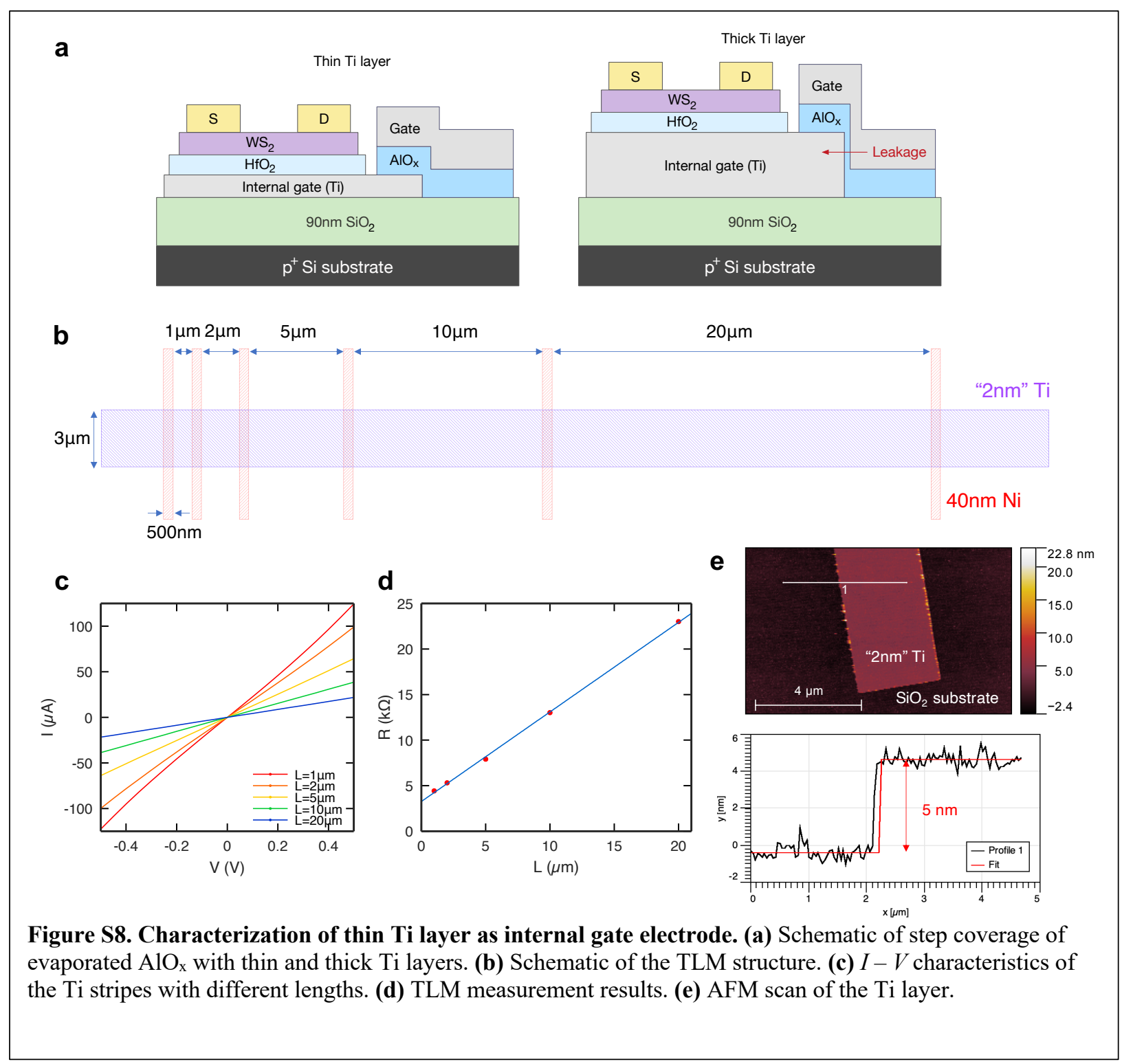

\section{Gate leakage current during $I_{D}-V_{G}$ scan}

Fig. S9 shows the gate leakage current $I_{G}$ as a function of $V_{G}$ during $I_{D}-V_{G}$ scan. We have also plotted the simulated $I_{G}-V_{G}$ curve from the RC network simulation in the figure, which shows very good agreement with the experiment. From the figure, we can see that the points at which the leakage current changes polarity coincide with the points at which $V_{G}-V_{\text {int }}$ changes polarity, as predicted in the simulation shown in Fig. S1d. 

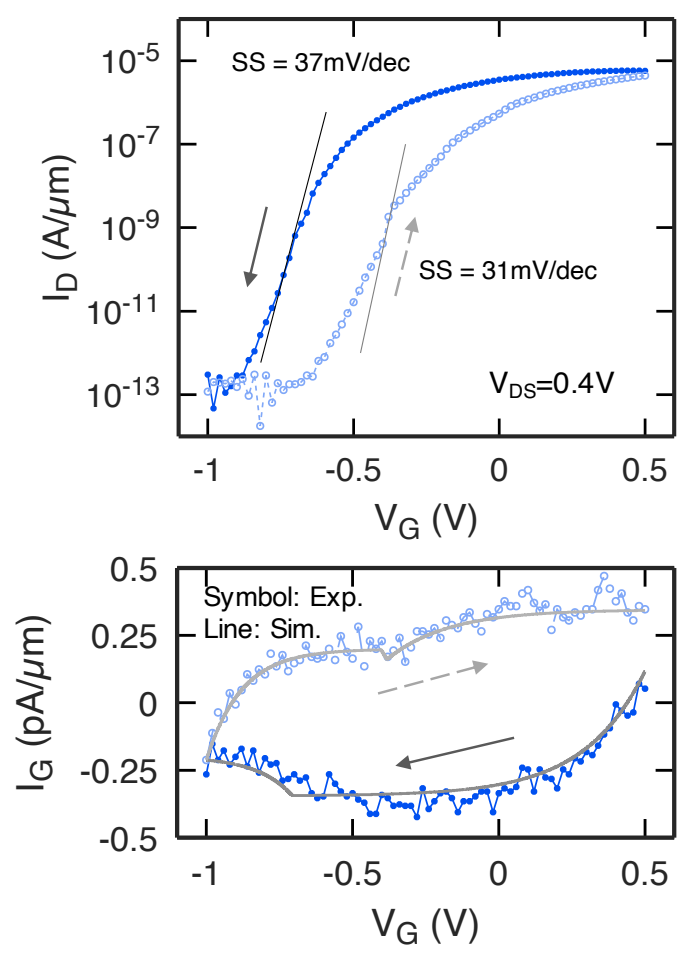

Figure S9. Measured and simulated $I_{G}-V_{G}$ during $I_{D}-V_{G}$ scan.

\section{References:}

1. Lu, C.-C.; Lin, Y.-C.; Yeh, C.-H.; Huang, J.-C.; Chiu, P.-W. High Mobility Flexible Graphene Field-Effect Transistors with Self-Healing Gate Dielectrics. ACS Nano 2012, 6, 4469-4474.

2. Yeh, C.-H.; Lain, Y.-W.; Chiu, Y.-C.; Liao, C.-H.; Moyano, D. R.; Hsu, S. S. H.; Chiu, P.-W.

Gigahertz Flexible Graphene Transistors for Microwave Integrated Circuits. ACS Nano 2014, 8, $7663-7670$.

3. English, C. D.; Smithe, K. H.; Xu, R.; Pop, E. Approaching Ballistic Transport in Monolayer $\mathrm{MoS}_{2}$ Transistors with Self-Aligned $10 \mathrm{~nm}$ Top Gates, In IEEE International Electron Devices Meeting (IEDM), San Francisco, CA, USA, Dec 2016; IEEE, 2016; pp 5.6.1-5.6.4 DOI: 10.1109/IEDM.2016.7838355.

4. Guerriero, E.; Pedrinazzi, P.; Mansouri, A.; Habibpour, O.; Winters, M.; Rorsman, N.; Behnam, A.; Carrion, E. A.; Pesquera, A.; Centeno, A.; Zurutuza, A.; Pop, E.; Zirath, H.; Sordan, R. HighGain Graphene Transistors with a Thin $\mathrm{AlO}_{\mathrm{x}}$ Top-Gate Oxide. Sci. Rep. 2017, 7, 2419. 
5. Penumatcha, A. V.; Salazar, R. B.; Appenzeller, J. Analysing Black Phosphorus Transistors Using an Analytic Schottky Barrier MOSFET Model. Nat. Commun. 2015, 6, 8948.

6. Appenzeller, J.; Zhang, F.; Das, S.; Knoch, J. Transition Metal Dichalcogenide Schottky Barrier Transistors - A Device Analysis and Material Comparison. In 2D Materials for Nanoelectronics, (Editors: M. Houssa, T. Dimoulas and A. Molle), CRC Press, Taylor \& Francis Group, Boca

Raton, FL, USA, 2016; Chapter 8, pp 207-240. 